\title{
Fluid Transport Properties from 3D Tomographic Images of Electrospun Carbon Electrodes for Flow Batteries
}

\author{
M. D. R. Kok ${ }^{\mathrm{a}}$, R. Jervis ${ }^{\mathrm{b}}$, P. R. Shearing ${ }^{\mathrm{b}}$, J.T. Gostick ${ }^{\mathrm{c}}$ \\ a Department of Chemical Engineering, McGill University, Montreal, Quebec, Canada \\ ${ }^{\mathrm{b}}$ Department of Chemical Engineering, University College London, London, England, UK \\ ${ }^{c}$ Department of Chemical Engineering, University of Waterloo, Waterloo, Ontario, Canada
}

\begin{abstract}
Three-dimensional $\mathrm{x}$-ray computer tomography images were obtained of electrospun poly(acrylonitrile) electrodes for a flow battery. The materials were imaged before and after carbonization. Information about the internal morphology; local fiber size and porosity, was analyzed and provided key insights into both the electrospinning and carbonizing processes. It was found that traditional imaging techniques may not be suitable for materials generated through electrospinning as it is a highly dynamic process. The fiber size tended to vary throughout the process while the porosity was relatively constant. Viscous flow was modelled through the material using the Lattice Boltzmann Method and the 3D flow fields that resulted provided further information about the role of heterogenous features on the performance of an electrospun electrode in a flow battery. The local porosity of the material had the largest effect on the material's flow dynamics.
\end{abstract}

\section{Introduction}

Porous media forms the backbone of a wide variety of crucial technologies ranging from the traditional water and air filtration membranes, to more specialized biomedical applications and high tech electrochemical systems. Fibrous media, a subset of porous media, are responsible for a significant share of this technology. Electrospinning, a method of preparing fibrous media by which nano and micro fibers are 'spun' from a solution using a large voltage potential $(>10 \mathrm{kV})$, has become probably the most common means of producing materials with customized structures. The main appeal of electrospinning, aside from the simplicity of the equipment required, is the tunability of the method (1), allowing the generation of fibers from as small as tens of nanometers to several microns with a wide array of morphologies, makes them ideal for a variety of applications. In biomedical applications recent work has utilized electrospun poly(caprolactone)/gelatin composite materials for guided bone regeneration (2), drug delivery in soft tissue $(3,4)$. Electrospun wound dressings are also being vigorously researched using poly (ether imide) (5), poly (lactic acid) (6), and a variety of other polymers (7-9). In electrochemistry they are being employed in a range of devices, such as high performance Lithium Ion battery separators (10-13), electrospun carbon nanofibers are also being produced as anode materials $(14,15)$, and gas diffusion layer in polymer electrolyte membrane fuel cells (16).

Flow batteries store and release energy by pumping a redox-active solution, stored in external tanks, through an electrochemical cell containing porous carbon fiber materials that act as both the diffusion medium and the electrode surface for electrochemical reaction. They are currently seen as potential solutions to grid-scale energy storage due to their reliability, relatively cheap and simple components, and the decoupling of power (determined by the size of the cell) and energy (determined by the volume of electrolyte) which makes them flexible to the needs of 
many different systems. (17) Electrospun materials have been proposed for use in flow batteries as a highly customizable electrode (18) and has been demonstrated to work effectively as one (19).

The advantages of electrospun materials is their small fibers (usually $<1 \mathrm{um}$ ), but this creates a significant issue for their characterization and the ability to model systems employing them. Even the routine step of quantifying fiber sizes requires analysis with a scanning electron microscope (SEM) images. Other key properties, such as porosity present similar difficulties. At best the porosity of an electrospun material can be estimated over an entire sample thickness, though locational variations can be observed by measuring small coupons take from a larger sheet (20). It is thus quite challenging to obtain a full understanding of the distributions of properties within the material and how the distributions can affect the behavior of the material. X-ray computed tomography (XCT) is a powerful characterization method that allows visualization of the physical structure of 3-d material's internal microstructure. XCT can provide quantitative parameters and fully resolved images of the microstructure for computer modelling, and has been employed with great success in the characterization of electrochemical systems such as batteries (21-25), supercapacitors (26), solid oxide fuel cells (27-33) and PEM fuel cells (34-37). Recently, XTC has also been applied to commercially available flow battery electrode materials $(38-40)$.

This work presents the first $\mathrm{x}$-ray computed tomographic images of electrospun poly(acrylonitrile) before and after carbonization into electrodes, at exceptional resolutions of 360 $\mathrm{nm}$ per voxel edge, allowing properties such as porosity and fiber diameter to be accurately determined throughout the whole sample despite the sub-micron feature size. Tomography in conjunction with high performance computing has allowed the first complete analysis of the internal structures and morphology of an electrospun material. Using structural images from XCT with computational fluid dynamic modelling we have studied the complex flow through electrospun materials and obtained information on velocity and pressure distributions, areas of stagnation and other complex flow properties present in inhomogeneous materials without relying on continuum mechanics. The present work demonstrates the ability of XCT to capture sufficient details over a sufficient large field-of-view to obtain meaningful information about the impact of the complex structure on flow behavior, and represent a significant step in the understanding and modelling of the transport process taking place in a flow battery electrode and their further optimization.

\section{Material Production}

\section{Methodology}

The electrospun materials were generated in-house on a custom-made system. The spin dope was pumped by a syringe pump, through a tube connected to a grounded needle. Throughout the duration of the electrospinning the needle was rastered on a linear motion actuator and a 4-inch rotating drum collector was used. Together these precautions ensured consistent material properties across a relatively large sample. The power was supplied by a negative polarity power supply (Glassman, MJ20N0400-11) which was connected to the drum to create the high voltage difference necessary for electrospinning. (41) The spin dope was pumped at a rate of $0.5-0.8 \mathrm{~mL} / \mathrm{hr}$ through a 16-gauge stainless steel needle placed $15 \mathrm{~cm}$ from the collector which was rotating at 5 $\mathrm{m} / \mathrm{min}$. The needle was rastered at a slow speed of $5 \mathrm{~mm} / \mathrm{s}$. The collector was held at a potential of $-15 \mathrm{kV}$.

The spin dope consisted of polyacrylonitrile (PAN) (Sigma, MW 150,000) dissolved at 12$13 \mathrm{wt} \%$ in anhydrous N,N-dimethylformamide (Sigma, 99.8\%). This range of concentrations was 
specifically chosen to generate materials to be used as flow battery electrodes, as such it was desired to make larger fibers than are typically achieved with electrospinning. It has been shown that the optimal fiber diameter for a porous flow battery electrode should be in the 1-2 $\mu \mathrm{m}$ range (18) which is 3-5x larger than the usual range for electrospun PAN (1). For electrospun materials, the largest parameter affecting the size of the fibers is the concentration of polymer in the spin dope. 12-13 wt\% is the maximum concentration of PAN in DMF that can effectively be pumped and electrospun; at higher concentrations pumping becomes untenable and the electrospinning process becomes very unstable.

Some of the produced materials were carbonized in a reducing furnace to create electrically conductive materials suitable for flow battery electrodes. (19) The material was first stabilized in air, with a heating rate of $5^{\circ} \mathrm{C} / \mathrm{min}$ and a plateau at $250^{\circ} \mathrm{C}$ for $75 \mathrm{~min}$. Argon was introduced at 25 sccm after the sample has been stabilized for an hour. The temperature was continually increased at a rate of $5^{\circ} \mathrm{C} / \mathrm{min}$, plateauing at $850^{\circ} \mathrm{C}$ and $1050^{\circ} \mathrm{C}$ both for $40 \mathrm{~min}$. During the carbonization process the materials are held under slight compression between two ceramic plates. More details about the material production steps can be found elsewhere. (19)

Tomography

Imaging of the electrospun materials was conducted using a Zeiss Xradia Versa 520 microCT machine (Carl Zeiss XRM, Pleasanton, CA), operating with a low source voltage of either 30 or $40 \mathrm{kV}$. It was found that a low source voltage and a sample diameter of $1 \mathrm{~mm}$ or less was required to obtain a good quality image due to the highly porous and low-z nature of the materials. 16012201 projections of $30 \mathrm{~s}$ exposure each were recorded through an angle of $360^{\circ}$ and reconstruction of the X-ray transmission images was conducted using a filtered back-projection reconstruction algorithm. Use of a 20X objective lens and binning of 1 yielded a voxel size of $0.38-0.40 \mu \mathrm{m}$, depending on individual scan setup. The grey-scale reconstructed volume is then segmented into a binary image using Avizo Fire software (FEI VSG, Mérignac Cedex France) to designate pixels as either 'fiber' or 'pore' materials, based on their grey-scale value. These binarised data sets are then exported as .tiff stacks and used as the structures for the computer modelling.

Lattice Boltzmann Method

Viscous pressure driven flow was simulated in-plane using the Lattice Boltzmann Method (LBM). This numerical model was implemented in the open-source LB solver Palabos, (42) using a 3D D3Q19 single relaxation time model with the standard Bhatnagar-Gross-Krook (BGK) collision operator. The initial conditions held the velocity at zero everywhere and fluid movement was induced by applying a fixed pressure gradient between the inlet and the outlet. (43) Convergence was determined by the standard deviation of the average energy of the system reaching $10^{-6}$. To ensure Stokes flow $(\operatorname{Re}<<1)$ the simulation was performed with pressure gradients ranging over several orders of magnitude and results outside this range were discarded. For true Stokes flow the material permeability of the system will not change for varying pressure. Further confirmation was achieved by determining the Reynold's number for the velocity distribution; in all cases the $\operatorname{Re}<10^{-2}$ was achieved.

Material Structural Properties

All computational analysis was performed in Python on the binarized tiff stacks obtained from the processing of the XCT data. Extracting structural features from tomography images, such as fiber diameter, porosity, pore size distributions, and so on, is the first level of information that one typically obtains. A number of commercial and open-sources packages are available for this, but it is still often necessary to devise custom methods for specific materials. In this work, the 
local fiber diameter distribution was of interest, so two similar schemes were developed. For both approaches, the first step is performing a Euclidian Distance Transform (EDT) on the fiber phase. Figure 1 shows the first two steps. The left shows a binary slice at an Z-position near the center of the stack, with ones (white) representing the fiber space and zeros (black) representing the pore space. The right image shows the results of a distance transform on the fibers. It should be noted that the 2D images and the analysis presented are just extracted from the 3D structure and all operations were conducted in $3 \mathrm{D}$.
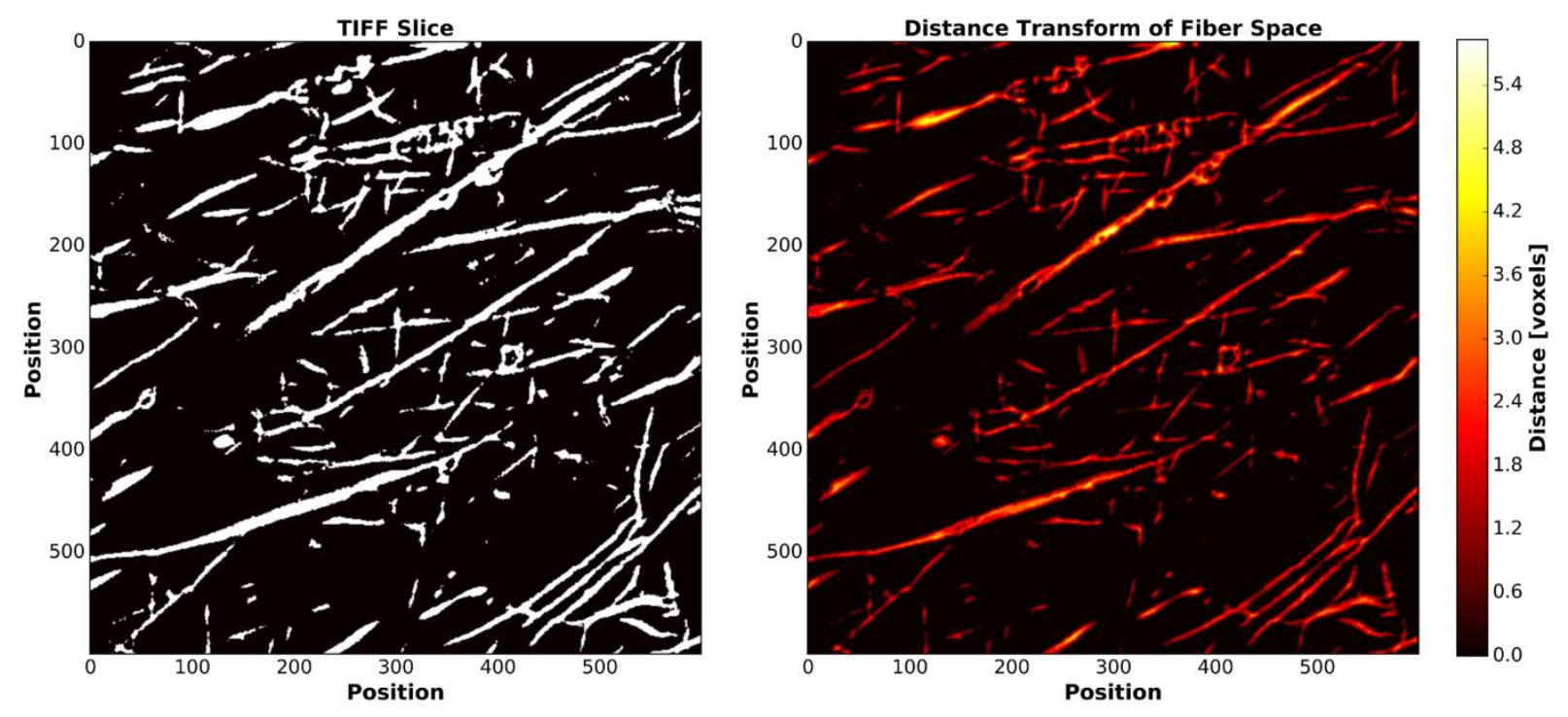

Figure 1. (Left) TIFF slice showing fibers in white and pore space in black, (Right) Distance Transform in Fiber Space, the numerical values are the distance from each fiber voxel to the nearest pore voxel, approximating the radius of a cylinder

Assuming the fibers are cylindrical, the peak values of the distance transform are a good approximation of the local radius. Peak values were found by applying a maximum filter distance transform. A maximum filter replaces the value stored in each voxel with the largest value in its neighborhood (defined by the structuring element), in theory replacing each voxel with the local radius. The results of this filter can be seen on the left side of Figure 2.

Averaging the value of every non-zero voxel will give an estimation of the average fiber radius in that plane. This value will be volume averaged however. In theory, significantly larger fibers are disproportionally represented and can skew the results. To confirm the data was not being skewed the second method was developed, where the single voxel thick 'skeleton' of the fibers was found. The value of the maximal filtered transform at this point would give the fiber averaged radius as opposed to the volume averaged value. An example of this 'skeletonized' radius is presented on the right side of Figure 2. After processing data with both methods, it was found the results were very similar and there was no evidence of skewing. This is likely because while there is a distribution in fiber sizes, it is unlikely that you would find a fiber that is several times larger than the average, large enough to alter the distribution. The volume averaged method is used in the data presented below because determining the 'skeleton' of the material is computationally intensive. 

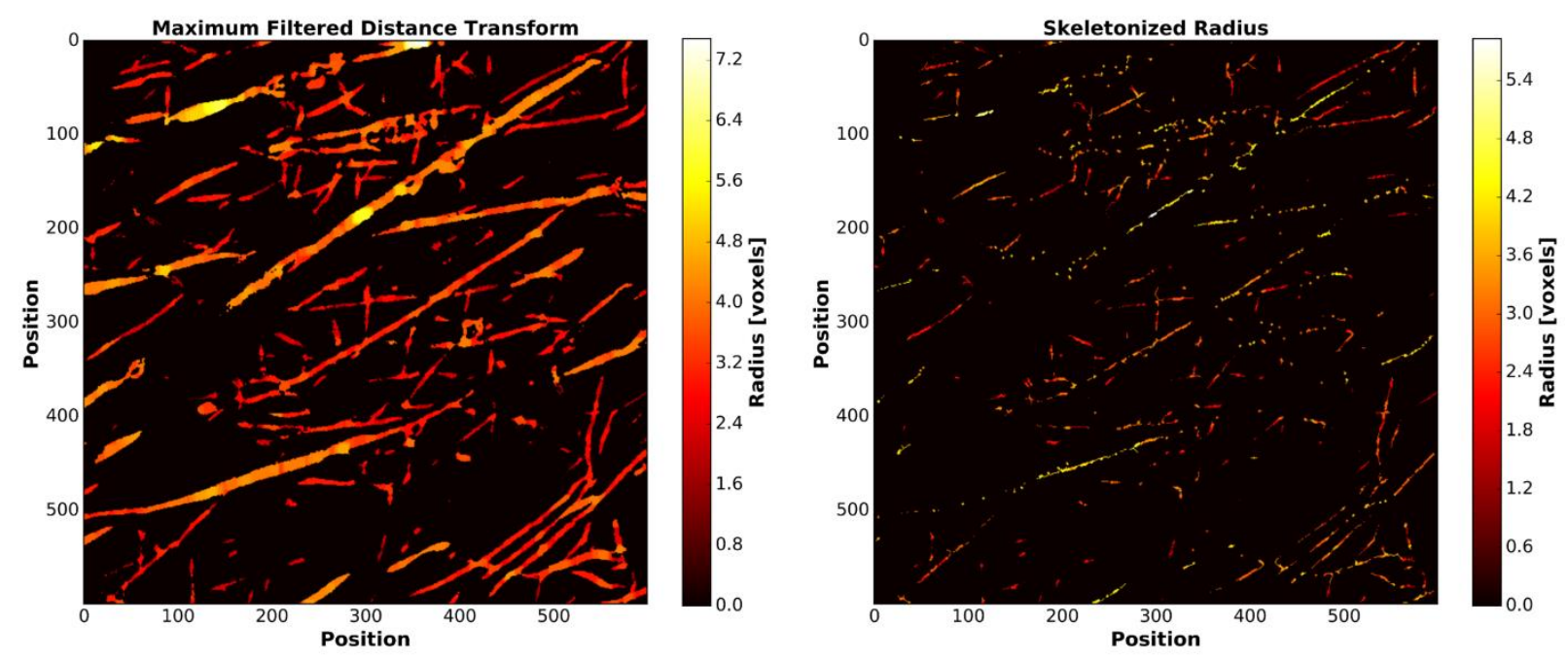

Figure 2. (Left) The result of passing a maximal filter over the distance transform data from Figure 1. (Right) The 'skeletonized' fiber radius.

The overall sample porosity is obtained by calculating the fraction of the voxels assigned to the pore space to the total voxels in the sample. In this work, the spatial distribution of porosity was also of interest. This is obtained by summing all the void voxels in a given plane and dividing by the full size of the plane. Although the porosity of a plane is actually an area fraction, in the case of voxels each plane represents a $400 \mathrm{~nm}$ thick subsection.

Permeability

The LBM simulation's output was a velocity field ( $\mathrm{x}, \mathrm{y}$ and $\mathrm{z}$ components), from which the Darcy permeability could easily be determined in both lattice and real units. To convert the velocities from lattice to real units we take advantage of the non-dimensionality of Reynold's Number; which should be equal in both domains:

$$
R e=\frac{\vec{u}_{L} L_{L}}{v_{L}}=\frac{\vec{u} L}{v}
$$

where $\vec{u}$ is the velocity vector, $v$ is the kinematic viscosity, the subscript $L$ denotes the lattice domain and no subscript indicates the physical domain. Rearranging gives:

$$
\vec{u}=\frac{v}{v_{L}}\left(\frac{L_{L}}{L}\right) u_{L}
$$

where $\left(\frac{L_{L}}{L}\right)$ is the reciprocal of the lattice distance and $v$ is the kinematic viscosity of the fluid, taken to be $1.004\left[\mathrm{~m}^{2} / \mathrm{s}\right]$ or equivalent to water at $20^{\circ} \mathrm{C}$ and $v_{L}$ was taken as 0.16667 (As recommended by the developers of Palabos). The lattice permeability can also be found, which is a perfect analogue to Darcy's Law in real units:

$$
\vec{q}=-\frac{\kappa}{\mu} \vec{\nabla} P
$$


where, $\vec{q}$ is the flux of fluid through the material $\left[\frac{\mathrm{m}^{3}}{\mathrm{~m}^{2} \cdot s}\right], \mu$ is the dynamic viscosity of the fluid $\left[\frac{\mathrm{kg}}{\mathrm{m} \cdot \mathrm{s}}\right]$, and $\vec{\nabla} P$ is the pressure gradient $\left[\frac{P a}{\mathrm{~m}}\right]$. The flux is determined by taking the average velocity in the direction of flow throughout the material. Every voxel has side lengths of 1 lattice unit, so in this case the average velocity is equal to the average flux without the need for normalizing. The pressure gradient and the viscosity are provided by the user before operation and are therefore known. It should be noted that technically the user specifies $v$, the kinematic viscosity and the conversion is made assuming the density is unity.

\section{Results and Discussion}

The data presented here analyses 3D tomography images of four samples, representing PAN concentrations of 12 and $13 \mathrm{wt} \%$ in the spin dope, before and after carbonization of the materials. Figure 3 is a sample representation of the domains modelled and analyzed throughout the results and discussion. The orientation of the axes will be consistent throughout the analysis. An axis averaged value (presented in the analysis below) represents the average of a value moving through that axis. The $\mathrm{x}$-axis shows the electrospinning process through time as materials are added slowly to build up the thickness of the mat. From a material perspective, the $\mathrm{z}$ and $\mathrm{y}$ axes are the same, one represents the rotation of the drum while the other is varied according to the rastering of the linear motion actuator, respectively. Since they were both moving relatively slowing no anisotropy was present in these directions, however they should ensure even distribution of properties. These two directions represent fibers in plane with the collector and will be referred to collectively as the collector plane. For the modelling, the z-axis is parallel to the direction of flow of the electrolyte in the medium and this corresponds to the direction perpendicular to the channels in a redox flow battery (shown in Figure 3 as streamlines generated in by LBM) and the y-axis corresponds to the direction parallel to the channels.

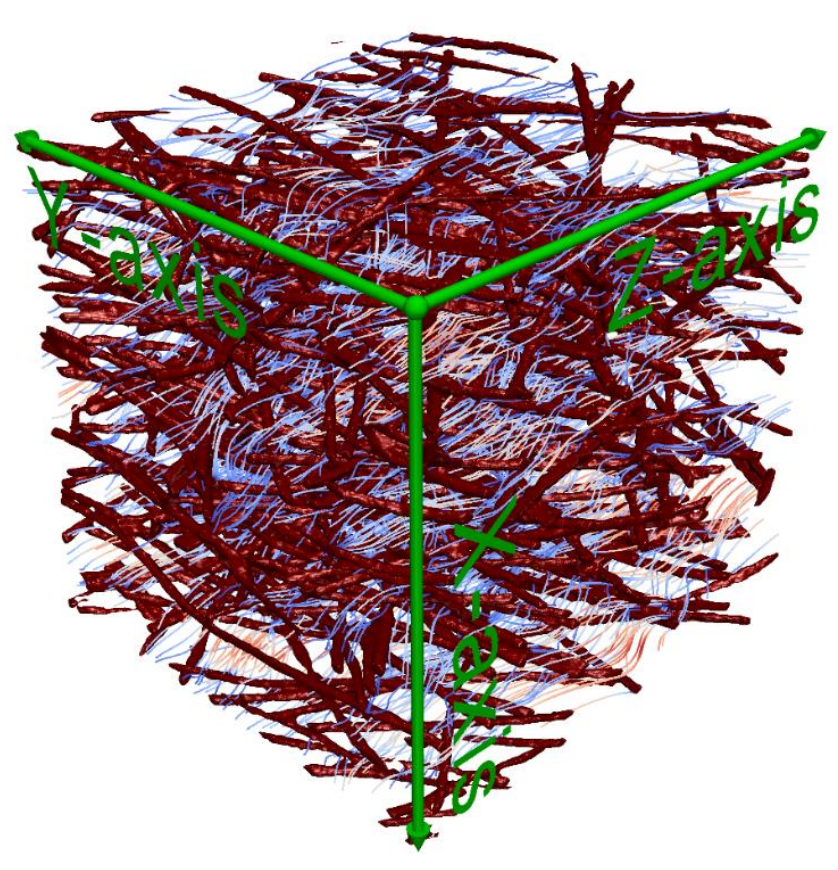


Figure 3. Representation of the modelled domain. Streamlines obtained through LBM show the direction of flow (z-axis). The $\mathrm{x}$-axis represents the electrospinning time while the $\mathrm{y}$-axis is across the domain.

\section{$\underline{\text { Fiber Diameter }}$}

A key challenge presented by analyzing any fibrous or porous media is the inability to analyze properties in the core of the material. This is especially true for feature sizes, such as fiber or pore diameter. For micro and nanofibers, effective imaging can only be done using scanning electron microscopy (SEM) which is of course limited to visualizing only the outer surface of the material. Nonetheless, 2D images of the surface are still widely used to infer structural information about the bulk material. In general, this approach is at best flawed; however, for electrospun materials it can be entirely misleading. Due to the nature of electrospinning the 'top' and the 'bottom' of a sample represents the beginning and end of the electrospinning process. These are the periods of time that are least stable throughout the entire operation as they represent the 'startup' and 'shutdown' of the electrospinner. This problem is further exacerbated by carbonization, as the fibers at the surfaces are in contact with plates that may cause temperature gradients as well as subject the fibers to different forces than experienced throughout the bulk.
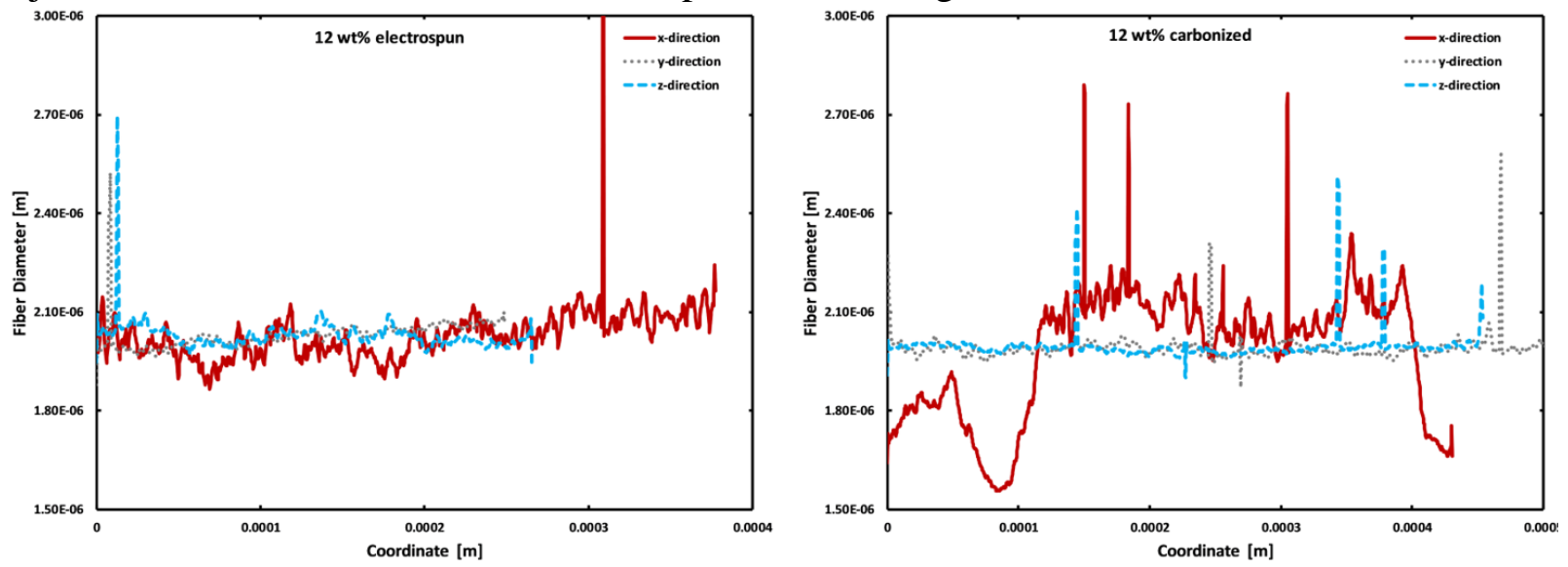

Figure 4. Fiber diameter distribution for all axes in a $12 \mathrm{wt} \%$ material. Left shows the distributions before the carbonization process. Right shows after carbonization.

Figure 4 (left) shows the fiber diameter distribution in a $12 \mathrm{wt} \%$ electrospun material before carbonization, obtained through XCT, and therefore representing the whole material. It should be noted that the sample volume was not perfectly cubic, and therefore the full lengths of the different dimensions are not equal. The electrospinning yielded relatively consistent fiber diameters in all dimensions. There is slightly more variation in the fiber diameter when averaged through electrospinning time (or sample thickness, $\mathrm{x}$-axis) as opposed to the other dimensions (collector plane), however it is relatively minor. Figure 4 suggests that estimating the fiber diameter from just the surface from just the surface information would be a relatively good estimation of the global fiber diameter in this case. This is not always true, however, as can be seen for the same material after carbonization. Figure 4 (right) shows that after carbonization the fiber diameter in collector plane remains relatively constant, but the variation through the thickness of the material (x-axis) is far from constant. At the extremes of the $\mathrm{x}$-dimension, the 'top' and 'bottom' of the samples, significant deviation from the average values are present. The fibers at the surfaces show a significant decrease in diameter when compared to the rest of the material. A likely explanation 
for this is the different conditions experienced at the surfaces compared to the bulk of the sample during the carbonization step. At the boundary, the material is in contact with the alumina plates, which could have created a temperature gradient through the material.

Although having smaller fibers towards the edges and larger fibers in the middle of a sample may not be a significant concern for a given application, from a characterization perspective this is a crucial difference. Analyzing this material after carbonization with SEM would result in an underestimation of the fiber diameter throughout the material by $25 \%$, leading to highly incorrect assumptions about key material properties such as solid surface area and permeability.

The $13 \mathrm{wt} \%$ materials do not show the same type of edge effects present in the $12 \mathrm{wt} \%$ materials; however, they further illustrate that insufficient information is obtained by only analyzing the outer surfaces of the materials. Figure 5 shows the fiber diameter distribution for the $13 \mathrm{wt} \%$ materials, with the electrospun materials shown on the left and the carbonized materials shown on the right. In this case, the fiber diameter is continually decreasing through the electrospinning process (x-dimension) due to uncontrollable variations in the spinning conditions. It is unclear why carbonization doesn't have the same effect in both samples. A possible explanation could be less compression or contact with the plates.
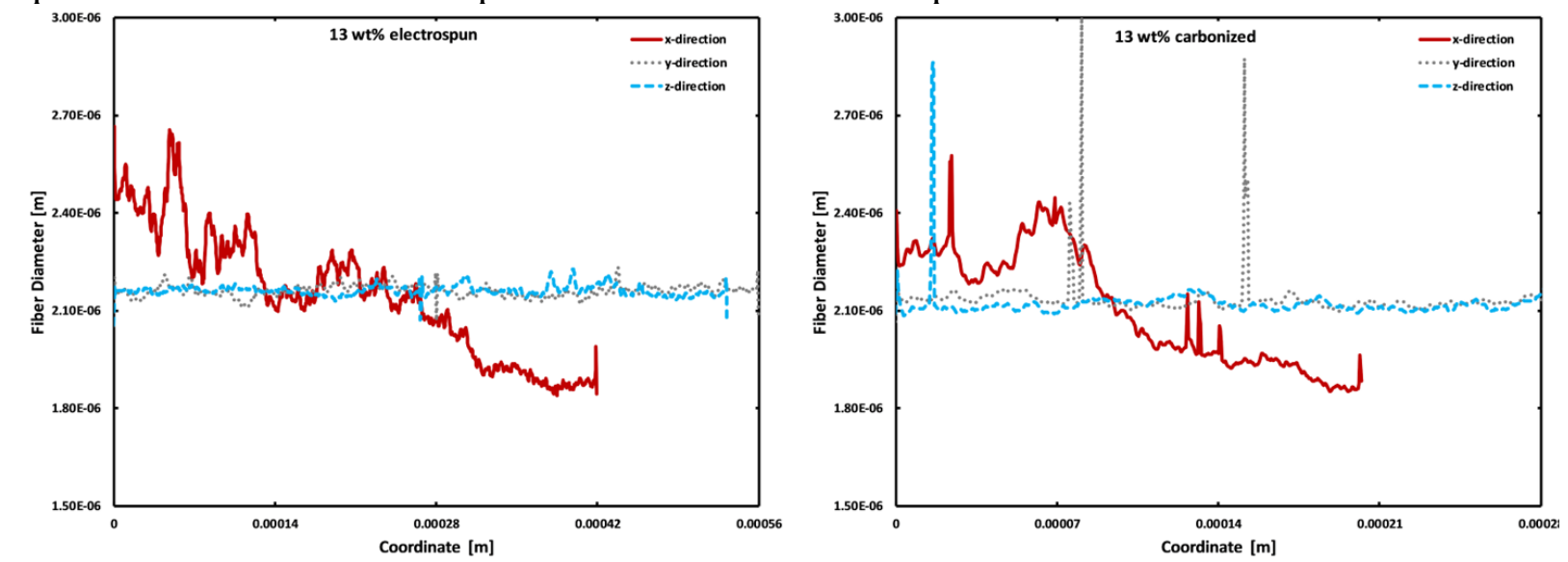

Figure 5. Fiber diameter distribution for all axes in a $13 \mathrm{wt} \%$ material. Left shows the distributions before the carbonization process. Right shows after carbonization

In general, Figure 4 and Figure 5 also demonstrate that the carbonizing process does not necessarily lead to the reduction in fiber diameter that is expected. In both the 12 and $13 \mathrm{wt} \%$ cases the carbonized material follows the overall scale as well as the shape of the electrospun material rather closely, excluding some edge effects.

\section{Porosity}

The distribution of porosity in the materials is much more straightforward. Figure 6 shows the porosity distribution in all dimensions for all samples. The 12 and $13 \mathrm{wt} \%$ materials are shown on the top and bottom, respectively, while the left side shows the electrospun materials before carbonization and the right side after carbonization. There are two main features to be shown in these distributions. Firstly, as was the case with the fiber diameters, much of the variability is in the $\mathrm{x}$-dimension, representing time throughout the electrospinning process. The even distributions in the collector plane $(\mathrm{y}, \mathrm{z})$ indicates the rotating drum collector and linear motion actuator were effective in ensuring an even distribution of fibers during the electrospinning process. Much like with the fiber diameters discussed in the previous section, the $12 \mathrm{wt} \%$ material doesn't exhibit a 
trend in either direction; there is some variability but it remains relatively constant throughout. On the other hand, the $13 \mathrm{wt} \%$ material has a general increasing trend in porosity that matches the decreasing fiber diameters shown in Figure 5. Assuming the rotating drum collector and the linear motion rastering perform ideally, a decreasing fiber diameter should lead to an increase in porosity, so the trend seen in the porosity of the $13 \mathrm{wt} \%$ materials is expected.
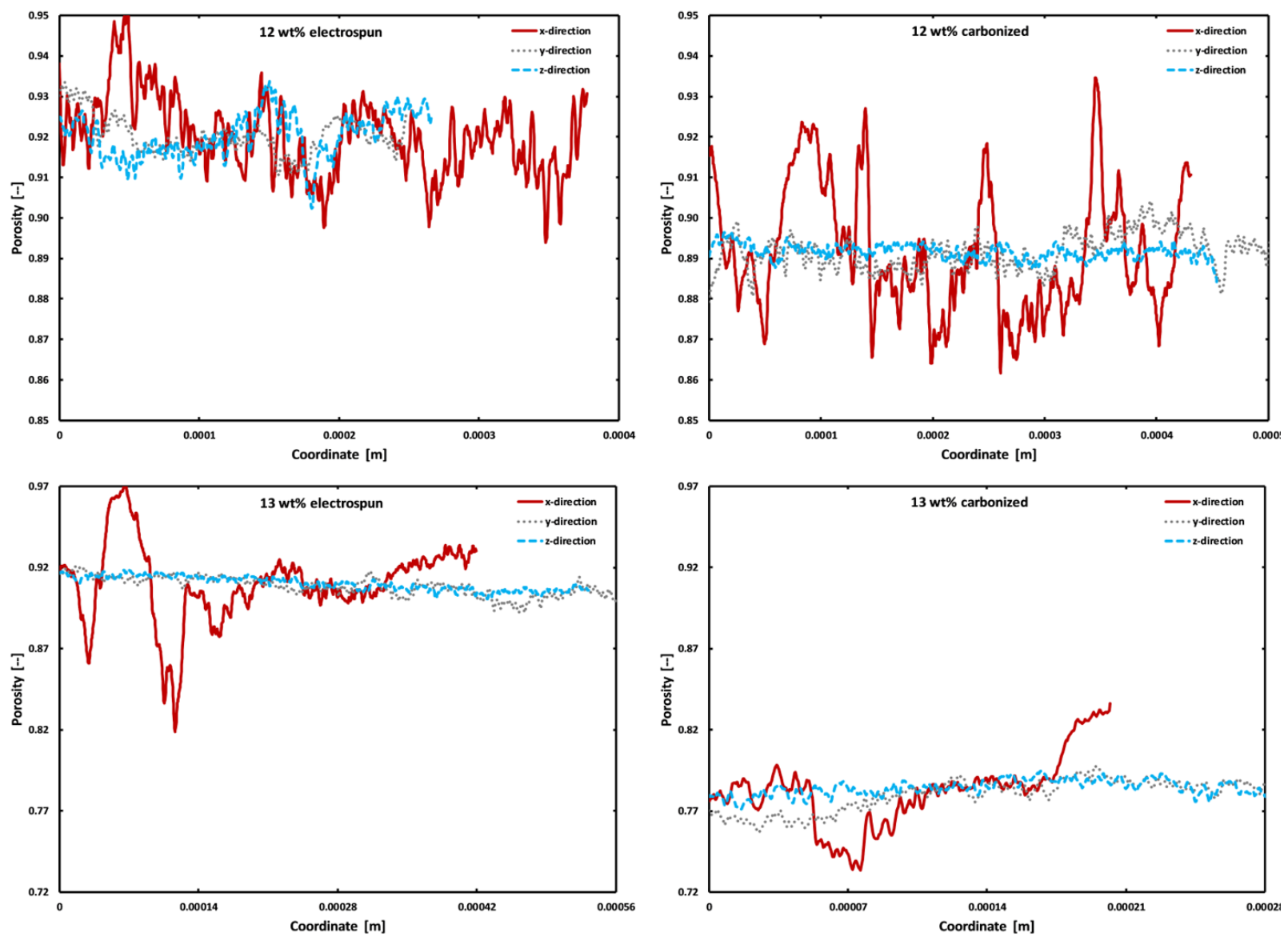

Figure 6. Porosity distribution for all samples. The top row shows $12 \mathrm{wt} \%$ data and the bottom row $13 \mathrm{wt} \%$ data. The electrospun samples are on the right and the carbonized materials are on the left.

The second observation that can be made from Figure 6 is that materials lose porosity during the carbonization process. (19) There are two possible explanations; firstly, the compression applied by the ceramic plates during the carbonization process leads to reduced porosity in the final material. Secondly, as materials are carbonized they lose mass. (19) If the fibers are remaining relatively constant in diameter, it's possible they are losing mass evenly throughout, causing them to contract and reducing the pore space between fibers.

Permeability

The LBM simulations allowed for the determination of the fluid velocity distribution on the pore level but also the determination of the Darcy permeability on the material level. Permeability is a key parameter for most porous media modelling studies, as it determines the material's resistance to flow and is often used as a 'continuum' property to fully describe the materials interactions with fluid flow. To validate the velocity distributions achieved through the 
LBM simulations, the material permeabilities determined through the simulations was compared to experimental data on the same materials (Figure 7). (19) The experimental results match the results determined through LBM quite closely. The reason for the relatively close data clustering of LBM results is that the materials were imaged under no compression and only one sample was imaged. By contrast the permeability measurements the modelling data is being compared to is done under a series of compressions.
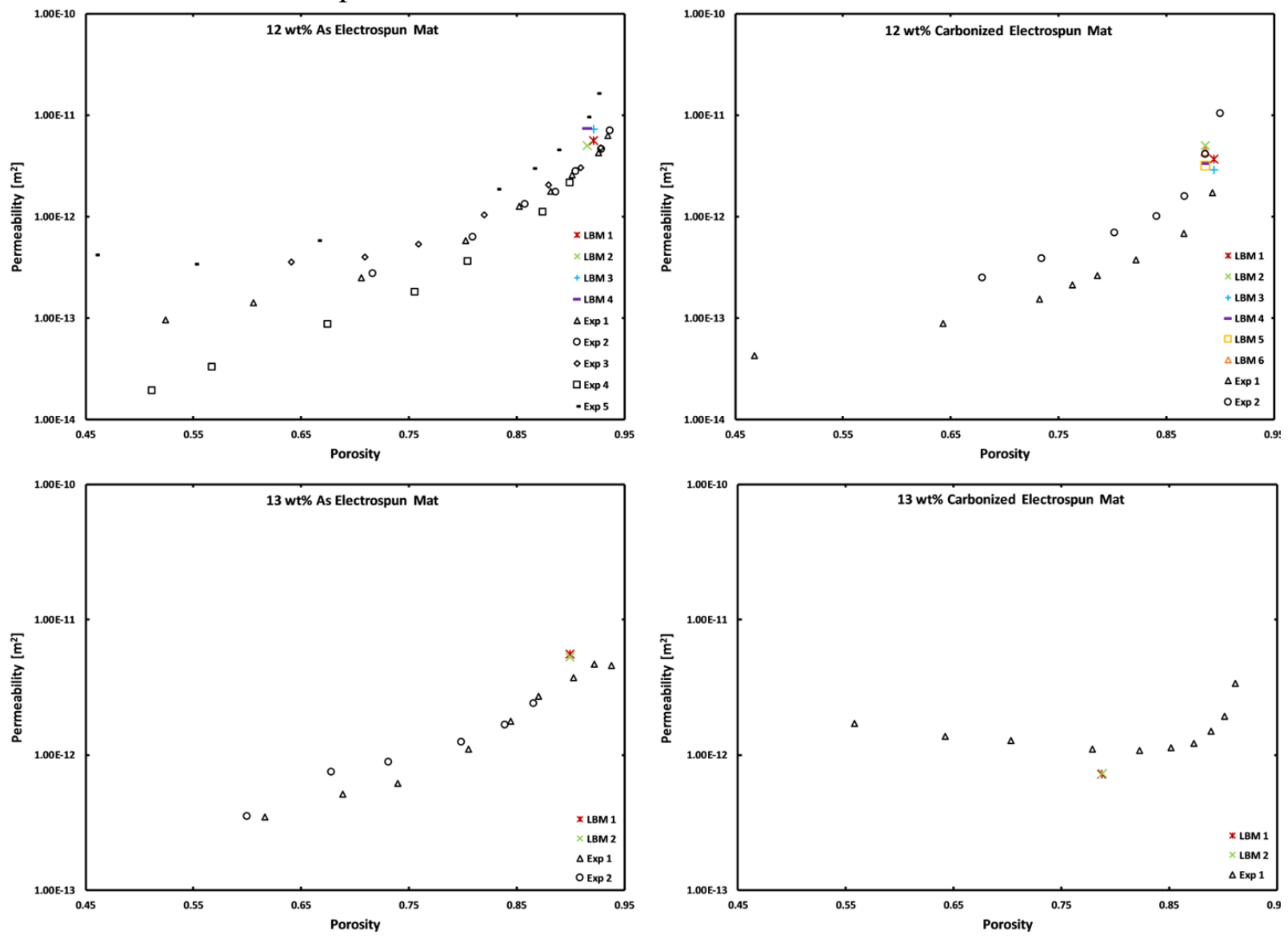

Figure 7. Comparison of Darcy Permeability determined through LBM with experimental data

Examining the velocity distributions in the materials provides further insights into the effects of fiber size and material porosity on permeability. The permeability distributions through the material were determined using the method described above, but on one plane at a time. When analyzed in this way it is important to note that the permeability distribution presented is more a 'contribution to permeability' distribution as permeability is by definition a continuum value without distributions especially on a pore scale. Plotting this distribution versus the fiber diameter and porosity in the x-dimension yields very telling results. Figure 8 shows the permeability distribution compared to both the porosity distribution on the left and fiber diameter distribution on the right. The data shown is the $\mathrm{x}$-axis of the $12 \mathrm{wt} \%$ carbonized material. The permeability clearly matches the porosity quite closely, there does seem to be some confounding effect of the fiber size but that is more likely an artifact of the highly coupled nature of fiber diameter and pore size in electrospun materials. 

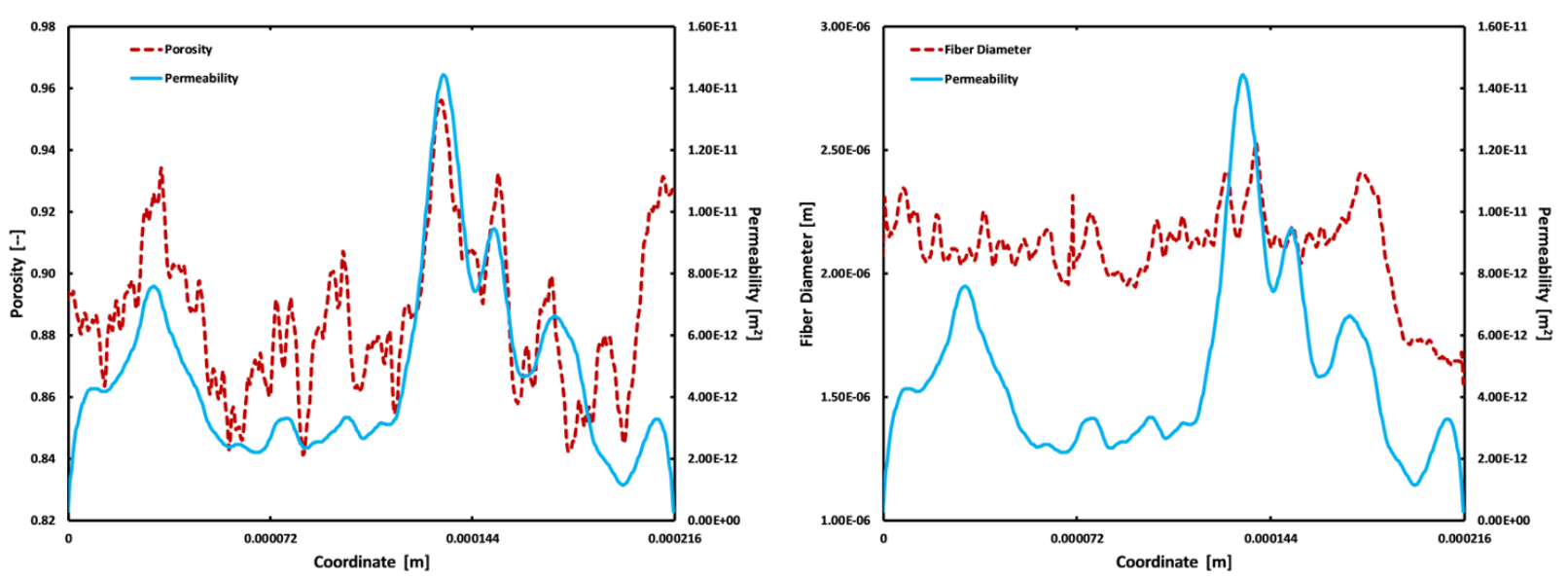

Figure 8. Permeability distribution versus (left) porosity and (right) fiber diameter. All in the xdimension

The permeability distribution obtained demonstrates the effect heterogeneity can have on the performance of these engineered materials. Small variations in fiber diameter and local porosity can double the material permeability, and these changes can happen on a scale of 10's of microns. Imaging these materials with high resolution XCT provides insights into actual flow that are not available through SEM or continuum modelling alone.

\section{Conclusions}

This paper explores the use of computational methods on 3D tomographical images of electrospun materials. To the best of our knowledge, this is the first time that electrospun fibers at this scale have been imaged in 3D using this technique. The materials imaged represent 12 and 13 wt\% PAN in DMF, both 'as spun' and after carbonization to make electronically conductive materials for electrochemical applications. Custom image analysis tools allowed for the determination of the fiber size and porosity distributions though the materials. It was found that for both properties the only significant variation was in the $\mathrm{x}$-dimension. Because this dimension corresponds to the electrospinning time, it provides direct evaluation of inconsistent or drifting production conditions. The other two dimensions show a very even distribution, which demonstrates the effectiveness of the rotating drum and the rastering of the electrospinning needle to ensure uniform properties.

The fiber diameter distributions showed two interesting characteristics that are both relevant to the common practice of determining material properties from SEM images. First, because electrospinning is a dynamic process, the fiber size at either surface can give a very poor representation of the fiber size throughout. The top and bottom represent the startup and shutdown of the process and are the least stable times. It was also shown that the size of the fibers can possibly change during the entirety of the spinning process. Which side is imaged under SEM can lead to very different ideas about material properties. The second characteristic relates to the carbonizing process. The $12 \mathrm{wt} \%$ material showed a very even distribution in fiber size before carbonization, but after the fiber size on the surfaces was significantly lower. The likely cause for this is compression or temperature gradients during the carbonization process which lead to higher conversion at the edges. This represents a significant issue because had this sample been analyzed with SEM the fiber size and therefore many material properties would be very incorrect and many assumptions about the carbonization process would be wildly misleading. 
The flow distribution in the materials was determined using the Lattice Boltzmann Method. This allowed for the determination of the material permeability which was compared to experimentally obtained results on the same materials imaged. The LBM permeabilities showed excellent agreement with the experimental results which validated the flow distributions. Further analysis was done on the distribution of permeability within the materials and it was shown quite clearly that the largest determining factor for permeability was the material porosity. The vital importance of using real-life structures, only obtainable through 3D methods such as XCT, to fully understand property distributions in modelling of porous media is highlighted in this study.

\section{References}

1. Kok MDR, Gostick JT. Transport properties of electrospun fibrous membranes with controlled anisotropy. J Membr Sci. 2015 Jan 1;473:237-44.

2. Ren K, Wang Y, Sun T, Yue W, Zhang H. Electrospun PCL/gelatin composite nanofiber structures for effective guided bone regeneration membranes. Mater Sci Eng C. 2017 Sep $1 ; 78: 324-32$.

3. Nadim A, Khorasani SN, Kharaziha M, Davoodi SM. Design and characterization of dexamethasone-loaded poly (glycerol sebacate)-poly caprolactone/gelatin scaffold by coaxial electro spinning for soft tissue engineering. Mater Sci Eng C. 2017 Sep 1;78:47-58.

4. Khalf A, Madihally SV. Modeling the permeability of multiaxial electrospun poly $(\varepsilon-$ caprolactone)-gelatin hybrid fibers for controlled doxycycline release. Mater Sci Eng C. 2017 Jul 1;76:161-70.

5. Haase T, Krost A, Sauter T, Kratz K, Peter J, Kamann S, et al. In vivo biocompatibility assessment of poly (ether imide) electrospun scaffolds. J Tissue Eng Regen Med. 2017 Apr $1 ; 11(4): 1034-44$.

6. Alavarse AC, de Oliveira Silva FW, Colque JT, da Silva VM, Prieto T, Venancio EC, et al. Tetracycline hydrochloride-loaded electrospun nanofibers mats based on PVA and chitosan for wound dressing. Mater Sci Eng C. 2017 Aug 1;77:271-81.

7. Zhang M, Lin H, Wang Y, Yang G, Zhao H, Sun D. Fabrication and durable antibacterial properties of 3D porous wet electrospun RCSC/PCL nanofibrous scaffold with silver nanoparticles. Appl Surf Sci. 2017 Aug 31;414:52-62.

8. Perumal G, Pappuru S, Chakraborty D, Maya Nandkumar A, Chand DK, Doble M. Synthesis and characterization of curcumin loaded PLA-Hyperbranched polyglycerol electrospun blend for wound dressing applications. Mater Sci Eng C. 2017 Jul 1;76:1196-204.

9. Zhou T, Sui B, Mo X, Sun J. Multifunctional and biomimetic fish collagen/bioactive glass nanofibers: fabrication, antibacterial activity and inducing skin regeneration in vitro and in vivo. Int J Nanomedicine. 2017;12:3495.

10. Ma X, Kolla P, Yang R, Wang Z, Zhao Y, Smirnova AL, et al. Electrospun polyacrylonitrile nanofibrous membranes with varied fiber diameters and different membrane porosities as lithium-ion battery separators. Electrochimica Acta. 2017 May 10;236:417-23. 
11. Freitag KM, Kirchhain H, Wüllen L van, Nilges T. Enhancement of Li Ion Conductivity by Electrospun Polymer Fibers and Direct Fabrication of Solvent-Free Separator Membranes for Li Ion Batteries. Inorg Chem. 2017 Feb 20;56(4):2100-7.

12. Ding Y, Hou H, Zhao Y, Zhu Z, Fong H. Electrospun polyimide nanofibers and their applications. Prog Polym Sci. 2016 Oct;61:67-103.

13. Yanilmaz M, Zhang $\mathrm{X}$. Polymethylmethacrylate/Polyacrylonitrile Membranes via Centrifugal Spinning as Separator in Li-Ion Batteries. Polym Basel. 2015;7(4):629-43.

14. Wu Y, Reddy MV, Chowdari BVR, Ramakrishna S. Long-Term Cycling Studies on Electrospun Carbon Nanofibers as Anode Material for Lithium Ion Batteries. ACS Appl Mater Interfaces. 2013 Nov 27;5(22):12175-84.

15. Kim D, Lee D, Kim J, Moon J. Electrospun Ni-Added SnO2-Carbon Nanofiber Composite Anode for High-Performance Lithium-Ion Batteries. ACS Appl Mater Interfaces. 2012 Oct 24;4(10):5408-15.

16. Chevalier S, Ge N, Lee J, George MG, Liu H, Shrestha P, et al. Novel electrospun gas diffusion layers for polymer electrolyte membrane fuel cells: Part II. In operando synchrotron imaging for microscale liquid water transport characterization. J Power Sources. 2017 Jun 1;352:281-90.

17. Wang WWW. Recent progress in redox flow battery research and development. Adv Funct Mater. 2013;23(8):970-86.

18. Kok MDR, Khalifa A, Gostick JT. Multiphysics Simulation of the Flow Battery Cathode: Cell Architecture and Electrode Optimization. J Electrochem Soc. 2016 Jan 1;163(7):A140819.

19. Liu S, Kok MDR, Kim Y, Barton JL, Brushett FR, Gostick JT. Evaluation of electrospun fibrous mats targeted for use as flow battery electrodes. under review.

20. Rashapov RR, Unno J, Gostick JT. Characterization of PEMFC Gas Diffusion Layer Porosity. J Electrochem Soc. 2015 Jan 1;162(6):F603-12.

21. Chen-Wiegart YK, Shearing P, Yuan Q, Tkachuk A, Wang J. 3D morphological evolution of Li-ion battery negative electrode LiVO2 during oxidation using X-ray nano-tomography. Electrochem Commun. 2012 Jul;21:58-61.

22. Cooper SJ, Eastwood DS, Gelb J, Damblanc G, Brett DJL, Bradley RS, et al. Image based modelling of microstructural heterogeneity in LiFePO4 electrodes for Li-ion batteries. $\mathrm{J}$ Power Sources. 2014 Feb 1;247:1033-9.

23. Di Michiel M, Merino JM, Fernandez-Carreiras D, Buslaps T, Honkimäki V, Falus P, et al. Fast microtomography using high energy synchrotron radiation. Rev Sci Instrum. 2005 Mar 18;76(4):043702. 
24. Finegan DP, Scheel M, Robinson JB, Tjaden B, Hunt I, Mason TJ, et al. In-operando highspeed tomography of lithium-ion batteries during thermal runaway. Nat Commun. 2015 Apr $28 ; 6: 6924$.

25. Finegan DP, Tudisco E, Scheel M, Robinson JB, Taiwo OO, Eastwood DS, et al. Quantifying Bulk Electrode Strain and Material Displacement within Lithium Batteries via High-Speed Operando Tomography and Digital Volume Correlation. Adv Sci. 2016 Mar 1;3(3):n/a-n/a.

26. Liu X, Jervis R, Maher RC, Villar-Garcia IJ, Naylor-Marlow M, Shearing PR, et al. 3DPrinted Structural Pseudocapacitors. Adv Mater Technol. 2016 Dec 1;1(9):n/a-n/a.

27. Nelson GJ, Harris WM, Lombardo JJ, Izzo Jr. JR, Chiu WKS, Tanasini P, et al. Comparison of SOFC cathode microstructure quantified using X-ray nanotomography and focused ion beam-scanning electron microscopy. Electrochem Commun. 2011 Jun;13(6):586-9.

28. Shearing PR, Bradley RS, Gelb J, Tariq F, Withers PJ, Brandon NP. Exploring microstructural changes associated with oxidation in Ni-YSZ SOFC electrodes using high resolution X-ray computed tomography. Solid State Ion. 2012 May 28;216:69-72.

29. Guan Y, Li W, Gong Y, Liu G, Zhang X, Chen J, et al. Analysis of the three-dimensional microstructure of a solid-oxide fuel cell anode using nano X-ray tomography. J Power Sources. 2011 Feb 15;196(4):1915-9.

30. Shearing PR, Bradley RS, Gelb J, Lee SN, Atkinson A, Withers PJ, et al. Using Synchrotron X-Ray Nano-CT to Characterize SOFC Electrode Microstructures in Three-Dimensions at Operating Temperature. Electrochem Solid-State Lett. 2011 Oct 1;14(10):B117-20.

31. Shearing PR, Gelb J, Yi J, Lee W-K, Drakopolous M, Brandon NP. Analysis of triple phase contact in Ni-YSZ microstructures using non-destructive $\mathrm{X}$-ray tomography with synchrotron radiation. Electrochem Commun. 2010 Aug;12(8):1021-4.

32. Izzo JR, Joshi AS, Grew KN, Chiu WKS, Tkachuk A, Wang SH, et al. Nondestructive Reconstruction and Analysis of SOFC Anodes Using X-ray Computed Tomography at Sub50 nm Resolution. J Electrochem Soc. 2008 May 1;155(5):B504-8.

33. Shearing PR, Gelb J, Brandon NP. X-ray nano computerised tomography of SOFC electrodes using a focused ion beam sample-preparation technique. J Eur Ceram Soc. 2010 Jun;30(8):1809-14.

34. Hartnig C, Manke I, Kuhn R, Kardjilov N, Banhart J, Lehnert W. Cross-sectional insight in the water evolution and transport in polymer electrolyte fuel cells. Appl Phys Lett. 2008 Mar 31;92(13):134106.

35. Hartnig C, Manke I, Kuhn R, Kleinau S, Goebbels J, Banhart J. High-resolution in-plane investigation of the water evolution and transport in PEM fuel cells. J Power Sources. 2009 Mar 15;188(2):468-74. 
36. Meyer Q, Mansor N, Iacoviello F, Cullen PL, Jervis R, Finegan D, et al. Investigation of Hot Pressed Polymer Electrolyte Fuel Cell Assemblies via X-ray Computed Tomography. Electrochimica Acta. 2017 Jul 10;242:125-36.

37. Manke I, Hartnig C, Grünerbel M, Lehnert W, Kardjilov N, Haibel A, et al. Investigation of water evolution and transport in fuel cells with high resolution synchrotron x-ray radiography. Appl Phys Lett. 2007 Apr 23;90(17):174105.

38. Jervis R, Brown LD, Neville TP, Millichamp J, Finegan DP, Heenan TMM, et al. Design of a miniature flow cell for in situ $\mathrm{x}$-ray imaging of redox flow batteries. J Phys Appl Phys. 2016;49(43):434002.

39. Brown LD, Neville TP, Jervis R, Mason TJ, Shearing PR, Brett DJL. The effect of felt compression on the performance and pressure drop of all-vanadium redox flow batteries. $\mathrm{J}$ Energy Storage. 2016 Nov 1;8:91-8.

40. Trogadas P, Taiwo OO, Tjaden B, Neville TP, Yun S, Parrondo J, et al. X-ray microtomography as a diagnostic tool for the electrode degradation in vanadium redox flow batteries. Electrochem Commun. 2014 Nov;48:155-9.

41. Barhate RS, Loong CK, Ramakrishna S. Preparation and characterization of nanofibrous filtering media. J Membr Sci. 2006 Oct 20;283(1-2):209-18.

42. Palabos [Internet]. FlowKit Ltd; Available from: http://www.palabos.org/

43. Degruyter W, Burgisser A, Bachmann O, Malaspinas O. Synchrotron X-ray microtomography and lattice Boltzmann simulations of gas flow through volcanic pumices. Geosphere. 2010 Oct 1;6(5):470-81. 